\title{
Good clinical practice in pulmonary embolism diagnosis: where do we stand today?
}

\author{
Marika Bajc • Lorenzo Maffioli • Massimo Miniati
}

Published online: 19 November 2013

(C) Springer-Verlag Berlin Heidelberg 2013

The intention of this editorial is to highlight ventilation/ perfusion single photon emission computed tomography (V/P SPECT) as an example of good clinical practice that may be used as an assessment tool during clinical auditing.

\section{Good clinical practice}

The basis of good practice is focused on patients. In nuclear medicine good practice includes: the procedure, the radiation dose and the results of therapy. Essential to optimal patient care is selecting the appropriate examination, based on knowledge of indications, advantages/limitations, complementary nature of other exams, risk/benefits and contraindication. All diagnostic examinations must be justified by the net benefit to the patient. Routinely performed diagnostic tests and treatments should be standardized according to internationally, nationally or locally agreed procedures.

Good clinical practice is a dynamic process which evolves with the general development of evidence-based medicine, equipment and techniques [1]. Evidence-based medicine provides the foundation on which guidelines for different procedures can be developed, which ideally can gain international acceptance.

\section{Bajc $(\bowtie)$}

Department of Clinical Physiology \& Nuclear Medicine, Lund University Hospital, 22185 Lund, Sweden

e-mail: Marika.bajc@med.lu.se

\section{Maffioli}

Nuclear Medicine Department, Ospedale Civile di Legnano, Via Papa Giovanni Paolo II, 20025 Legnano, Milan, Italy

M. Miniati

Department of Experimental and Clinical Medicine, University of Florence, 50134 Florence, Italy
The implementation of best practice procedures is, therefore, crucial for optimal outcome both in diagnostic tools (doses as low as possible to achieve quality) and treatment (minimum side-effects). Observation and recording include short-term results, such as correct diagnosis, side-effects and long-term assessment by follow-up procedures.

The criteria of good procedures (i.e. good practice) are thus the cornerstones for development of clinical audits; these should be the basis of assessments regardless of the type of the audit (external, internal, comprehensive or partial). Working also as a clinical auditor we have realized that the guidelines for some methods are not frequently implemented.

\section{Historical evolution of lung scintigraphy}

Historically, lung scintigraphy was the first method that made it possible to obtain spatial two-dimensional information on ventilation $(\mathrm{V})$ and perfusion $(\mathrm{P})$ in vivo. The single most important application of lung scintigraphy is in the evaluation of patients with suspected pulmonary embolism (PE). As early as 1964, Henry N. Wagner demonstrated the value of perfusion scintigraphy in the detection of PE [2]. Single-head gamma cameras were generally used to perform planar images. Planar imaging has now become obsolete, particularly when the issue is the identification and quantification of focal or regional aberration of organ function. Moreover, previous probabilistic interpretation criteria and the associated reporting methods, according to PIOPED or modified PIOPED, are unsatisfactory [3]. Using PISAPED criteria with clear answers, taking into account vascular anatomy of the lung has clearly improved diagnosis [4]. Efficient lung scintigraphic methods routinely incorporate ventilation studies. Using a dual-head camera, Palmer et al. developed a fast and efficient method for V/P SPECT for clinical practice with a total acquisition time of only $20 \mathrm{~min}$ [5]. Moreover, they developed an algorithm to calculate 
the quotient between ventilation and perfusion and to present it as V/P quotient images, thereby facilitating PE diagnosis and quantification of PE extension. This led to the use of the term quantitative V/P SPECT. The method was validated for diagnosis of $\mathrm{PE}$ by using a porcine model. This led to its adoption as the standard method, by confirming the superiority of tomography over planar imaging with excellent interobserver agreement in identifying perfusion defects at the subsegmental level [6]. The advantage of tomographic over planar images for PE detection was recently confirmed in human studies [7].

\section{Basis for good clinical practice: guidelines}

The new European guidelines recommend the holistic interpretation of lung SPECT $[8,9]$. Clinicians can only benefit from reports which clearly state the presence or absence of PE. Moreover, the combined evaluation of ventilation and perfusion abnormalities has improved the sensitivity of the method by identifying changes in lingula and middle lobe which is neither possible with planar images nor with perfusion SPECT only. Moreover, V/P SPECT permits one to identify patterns typical of other disorders such as chronic obstructive pulmonary disease (COPD), pneumonia and left heart failure. A broad V/P SPECT study showed that this is possible in the vast majority of cases [10].

These guidelines and recommendations provide general advice on the use of current clinically used method. They are intended to create standard protocols for the use and interpretation of V/P SPECT not only for PE diagnosis but also other pulmonary diseases with the aim of improving patient management by adopting clearly formulated reports.

\section{Outcome: crucial in clinical care}

According to good clinical practice the best available point of reference is adequate patient follow-up, i.e. in patients with suspected PE this means to prove normalization of perfusion or to identify recurrent PE. As V/P SPECT is applicable to all patients has no contraindications and low radiation exposure, it is ideal for follow-up to measure outcome. So, we possess the most valuable tool, that is, the possibility to assess the recovery of perfusion after acute PE. This aspect has some important implications from psychological to economical ones. Moreover, it offers the opportunity to tailor anticoagulant therapy (i.e. home vs hospital treatment). It gives us the possibility to adjust duration of therapy. V/P SPECT also holds further promise in predicting short-term outcome. Furthermore, follow-up is fundamental when dealing with PE patients who have known co-morbidities such as COPD or chronic left heart failure.

\section{Importance of ventilation scintigraphy}

PE diagnosis and other cardiopulmonary diseases

The ventilation scan maps regional ventilation and helps define lung borders, thereby facilitating the recognition of peripheral perfusion defects. This is very important for medial lobe and lingula where a perfusion scan is not sufficient. The ventilation scan may also provide additional information about cardiopulmonary disorders, other than PE. For example, in COPD, the distribution of ventilation is uneven and in aerosol studies focal deposition is often observed in central or peripheral airways. Pneumonia causes regional ventilation defects, usually more extensive than the associated perfusion defects. Combined ventilation and perfusion studies increase specificity for PE diagnosis and allow recognition of alternative pathology. Left heart failure is a further diagnosis that is often observed among patients with suspected PE. The typical pattern is anti-gravitational redistribution of perfusion. As ventilation is usually less redistributed than perfusion, V/P mismatch may be observed in dorsal regions. This V/P mismatch has a non-segmental pattern and should not be misinterpreted as PE. It is therefore recommended that in PE diagnosis a combined 1-day protocol is used $[8,9]$.

\section{Perfusion SPECT}

Perfusion SPECT is only recommended in emergencies when the diagnosis of $\mathrm{PE}$ would otherwise be missed as pointed out in a recent article and editorial $[11,12]$.

\section{Comparison with MDCT}

The absence of a satisfactory gold standard for PE diagnosis poses difficulties for the assessment of sensitivity, specificity and accuracy of all diagnostic methods. The best available standard is adequate follow-up of the patient for recurrence of $\mathrm{PE}$ or alternative diagnoses. The most rigorous study of multidetector computed tomography (MDCT) in PE diagnosis is the PIOPED II study, which showed an overall sensitivity for PE of $78 \%$ when non-diagnostic studies were included [13]. This led to the observation that the false-negative rate of $22 \%$ for MDCT indicates the need for additional information to rule out PE. In the PIOPED II study the positive predictive value for a PE within a lobar pulmonary artery was $97 \%$ but fell to $68 \%$ and $25 \%$ at the segmental and subsegmental levels, respectively. Bajc et al. and a prospective study by Gutte et al. showed that V/P SPECT has higher sensitivity and specificity and less non-diagnostic findings than MDCT [10, 14]. A recent study by Mahdavi et al. showed that in the presence of an indeterminate CT angiography (CTA) in patients with 
high clinical suspicion of PE, V/P SPECT often provides a diagnosis [15].

\section{Hybrid SPECT/CT}

It was stressed by Roach et al. that V/P SPECT/CT offers the potential for a single imaging procedure yielding high sensitivity and specificity for the detection of PE [16]. Hybrid imaging is a novel technique primarily developed to improve sensitivity and specificity of oncological diseases. We would not initially recommend it for PE diagnosis to all patients with suspected PE. It would not be in line with good clinical practice. The application of SPECT/CT might be interesting for COPD patients where, as an additional diagnosis, a small tumour might be identified. In addition it has the potential in assisting in the classification of COPD degree. This is currently under investigation.

\section{Importance of small PE}

The high sensitivity of V/P SPECT allows the detection of subsegmental perfusion defects. The clinical importance of identifying small perfusion defects is still a matter of dispute. It is argued that such small defects are irrelevant because on clinical short-term follow-up (3-6 months), no patients died. What we learned by following patients with PE over time is that patients with residual subsegmental PE after treatment have persistent risk factors for recurrence and might develop chronic PE [17]. The importance of diagnosing subsegmental $\mathrm{PE}$ has been recently underscored by showing that it is as dangerous as proximal PE [18]. Here again, V/P SPECT is an outstanding tool capable of showing small perfusion defects better than any other method. In fact, other methods used for PE diagnosis such as MDCT and MRI feature a low sensitivity at the subsegmental level $[13,19]$. This is the area that was not stressed enough in the European guidelines. An additional area open for clinical research is COPD, inasmuch as functional images can provide valuable insight into the heterogeneity of the disease. In addition to COPD, left heart failure is another interesting field to be explored.

After dealing with lung nuclear medicine diagnostic development over the last 15 years - we are still very excited — we would like to stress again the unique value of V/P SPECT as the only method that provides a functional image of the regional distribution of ventilation and perfusion. In this regard, V/P SPECT should not be taken as equivalent to MDCT. V/P SPECT should be the method of first choice for diagnosis of PE, due to the much lower radiation burden and applicability to all patients. It provides the basis for good practice: the relative simplicity of the procedure, the limited radiation dose and the possibility to assess the effect of therapy over time.
Therefore, practitioners should attempt to ensure that V/P SPECT becomes more widely available. It is our responsibility to implement tomographic methods and use the new interpretation criteria with definitive reporting and last but not the least to communicate daily with the clinicians so that the best technique is used in all patients. We are too slow in implementation of the method and learning and using its possibility. Though the wide acceptance of routine V/P SPECT imaging for diagnosing lung disease faces its challenges, in our experience, the simplicity and functional information that extends from this innovative diagnostic tool is creating new pathways towards early diagnoses, intervention and treatment of a range of pulmonary morbidities and comorbidities.

\section{Conclusion}

The crucial issue in good clinical practice is the outcome. For this purpose, V/P SPECT has its place as it fulfils the following basic requirements:

1. Fast procedure and prompt availability of results

2. Applicability to all patients

3. High diagnostic accuracy and few non-diagnostic reports

4. Low radiation dose

5. Utility for selection of treatment strategy

6. Suitability for follow-up and research studies

In the dynamic process of good clinical practice, clinical audits will work as a benchmarking tool to refine and improve the diagnostic criteria.

\section{References}

1. Soimakallio S, Alanen A, Järvinen H, Ahonen A, Ceder K, LyyraLaitinen T, et al. Clinical audit: development of the criteria of good practices. Radiat Prot Dosimetry 2011;147(1-2):30-3.

2. Wagner Jr HN. Pulmonary scanning. Northwest Med 1964;63: 857-64.

3. PIOPED Investigators. Value of the ventilation/perfusion scan in acute pulmonary embolism. Results of the prospective investigation of pulmonary embolism diagnosis (PIOPED). JAMA 1990;263(20): 2753-9.

4. Miniati M, Pistolesi M, Marini C, Di Ricco G, Formichi B, Prediletto $\mathrm{R}$, et al. Value of perfusion lung scan in the diagnosis of pulmonary embolism: results of the Prospective Investigative Study of Acute Pulmonary Embolism Diagnosis (PISA-PED). Am J Respir Crit Care Med 1996;154(5):1387-93.

5. Palmer J, Bitzén U, Jonson B, Bajc M. Comprehensive ventilation/ perfusion SPECT. J Nucl Med 2001;42(8):1288-94.

6. Bajc M, Bitzén U, Olsson B, de Sá Perez V, Palmer J, Jonson B. Lung ventilation/perfusion SPECT in the artificially embolized pig. J Nucl Med 2002;43(5):640-7.

7. Gutte H, Mortensen J, Jensen CV, von der Recke P, Petersen CL, Kristoffersen US, et al. Comparison of V/Q SPECT and planar V/Q 
lung scintigraphy in diagnosing acute pulmonary embolism. Nucl Med Commun 2010;31(1):82-6.

8. Bajc M, Neilly JB, Miniati M, Schuemichen C, Meignan M, Jonson B, EANM Committee. EANM guidelines for ventilation/perfusion scintigraphy: part 1. Pulmonary imaging with ventilation/perfusion single photon emission tomography. Eur J Nucl Med Mol Imaging 2009;36(8):1356-70.

9. Bajc M, Neilly JB, Miniati M, Schuemichen C, Meignan M, Jonson B. EANM guidelines for ventilation/perfusion scintigraphy: part 2 . Algorithms and clinical considerations for diagnosis of pulmonary emboli with V/P(SPECT) and MDCT. Eur J Nucl Med Mol Imaging 2009;36(9):1528-38.

10. Bajc M, Olsson B, Palmer J, Jonson B. Ventilation/perfusion SPECT for diagnostics of pulmonary embolism in clinical practice. J Intern Med 2008;264(4):379-87.

11. Bajc M, Miniati M, Jögi J, Stein PD. Perfusion SPECT in patients with suspected pulmonary embolism. Eur J Nucl Med Mol Imaging 2013;40(9):1432-7.

12. Schuemichen C. Perfusion SPECT in patients with suspected pulmonary embolism: how much sensitivity is needed to keep patients alive? Eur J Nucl Med Mol Imaging 2013;40(9):1428-31.

13. Stein PD, Fowler SE, Goodman LR, Gottschalk A, Hales CA, Hull $\mathrm{RD}$, et al. Multidetector computed tomography for acute pulmonary embolism. N Engl J Med 2006;354(22):2317-27.
14. Gutte H, Mortensen J, Jensen CV, Johnbeck CB, von der Recke P, Petersen CL, et al. Detection of pulmonary embolism with combined ventilation-perfusion SPECT and low-dose CT: head-to-head comparison with multidetector CT angiography. J Nucl Med 2009;50(12): 1987-92.

15. Mahdavi R, Caronia J, Fayyaz J, Panagopoulos G, Lessnau KD, Scharf SC, et al. Agreement between SPECT V/Q scan and CT angiography in patients with high clinical suspicion of PE. Ann Nucl Med 2013. doi:10.1007/s12149-013-0753-1.

16. Roach PJ, Schembri GP, Bailey DL. V/Q scanning using SPECT and SPECT/CT. J Nucl Med 2013;54(9):1588-96.

17. Alhadad A, Miniati M, Alhadad H, Gottsäter A, Bajc M. The value of tomographic ventilation/perfusion scintigraphy (V/PSPECT) for follow-up and prediction of recurrence in pulmonary embolism. Thromb Res 2012;130(6):877-81.

18. den Exter PL, van Es J, Klok FA, Kroft LJ, Kruip MJ, Kamphuisen PW, et al. Risk profile and clinical outcome of symptomatic subsegmental acute pulmonary embolism. Blood 2013;122(7): 1144-9.

19. Stein PD, Chenevert TL, Fowler SE, Goodman LR, Gottschalk A, Hales CA, et al. Gadolinium-enhanced magnetic resonance angiography for pulmonary embolism: a multicenter prospective study (PIOPED III). Ann Intern Med 2010;152(7):434-43. W142-3. 